\title{
Blazar Jets Perturbed by Magneto-Gravitational Stresses in Supermassive Binaries
}

\author{
A. Cavaliere ${ }^{1,2}$, M. Tavani ${ }^{1,2,3,4}$, V. Vittorini ${ }^{2}$
}

\begin{abstract}
We study particle acceleration and radiative processes in Blazar jets under recurring conditions set by gravitational perturbations in supermassive binary systems. We consider the action from a companion orbiting a primary black hole of $\sim 10^{8} M_{\odot}$, and perturbing its relativistic jet. We discuss how such conditions induce repetitive magneto-hydrodynamic stresses along the jet, and affect its inner electron acceleration and radiative processes. Specifically, we study how macroscopic perturbations related to increased jet "magnetization" end up into higher radiative outputs in the optical, X-ray and gamma-ray bands. We find first an increase in magnetic field strength as gauged in the optical band from the Synchrotron emission of electrons accelerated in kinetic processes stimulated by reconnecting magnetic lines. The energetic electrons then proceed to up-scatter the Synchrotron photons to $\mathrm{GeV}$ energies after the canonical Synchrotron-Self Compton radiation process. Our model implies a specific, recurring pattern in the optical to gamma-ray emissions, made of high peaks and wide troughs. Progressing accelerations caused by spreading reconnections will produce an additional Synchrotron keV component. Such outbursts provide a diagnostics for enhanced acceleration of electrons which can up-scatter photons into the TeV range. We discuss how our model applies to the BL Lac object PG 1553+113, arguably the best candidate to now for high amplitude, recurring modulations in its gamma-ray emissions. We also consider other BL Lacs showing correlated keV - TeV radiations such as Mrk 421.
\end{abstract}

Subject headings: gamma rays: observations - BL Lac Objects; individual: PG $1553+113$, Mrk 421.

\footnotetext{
${ }^{1}$ Astronomia, Accademia Nazionale dei Lincei, via della Lungara 10, I-00165 Roma, Italy

${ }^{2}$ INAF/IAPS-Roma, via del Fosso del Cavaliere 100, I-00133 Roma, Italy

${ }^{3}$ Universitá "Tor Vergata", Dipartimento di Fisica, via della Ricerca Scientifica 1, I-00133 Roma, Italy

${ }^{4}$ Gran Sasso Science Institute, viale Francesco Crispi 7, I-67100 L'Aquila, Italy
} 


\section{Introduction}

Blazars are singled out among the Active Galactic Nuclei (AGN) by their relativistically collimated jets with bulk Lorentz factors $\Gamma \sim 5-15$ (Urry \& Padovani 1995). They are launched by a central super-massive black hole (SMBH) with mass $M 10^{8} M_{\odot}$, and are Doppler-boosted when a jet happens to be closely aligned with our line of sight.

Blazars are extreme in several respects. The observed outputs are very bright, up to $10^{49} \mathrm{erg} / \mathrm{s}$ in isotropic extrapolation, and strongly variable on diverse timescales from years to minutes depending on the observed bands. They show highly non-thermal spectra with energy distributions (SEDs) constituted by two humps: one peaking in the $I R$-UV bands of clear Synchrotron (S) origin; the other extending from hard X to gamma rays, of likely inverse Compton (IC) nature (for basics see Rybicki \& Lightman 1979). Both are produced by highly relativistic electrons with random Lorentz factors up to $\gamma_{p} \sim 10^{3}$ that inhabit the jets.

Two main Blazar flavors are discerned (see Peterson 1997, Ghisellini 2016). The BL Lac-type sources feature two comparable spectral humps. The Flat Spectrum Radio Quasars (FSRQs), on the other hand, feature Compton-dominated spectra at gamma-ray energies, but also a conspicuous Big Blue Bump and the strong, broad optical emission lines common to many quasars; these thermal features yield evidence of a gas-rich environment surrounding the SMBH out to some $10^{-1}$ pc.

Relativistic electrons within the jet emit by synchrotron $(\mathrm{S})$ radiation an observed (and isotropically extrapolated) luminosity $L_{S} \propto n R^{3} B^{2} \gamma_{p}^{2} \Gamma^{4}$, in terms of the density $n$ of relativistic electrons within the source size $R$, and of the general magnetic field $B \sim 1 \mathrm{G}$ threading the jet. On the other hand, the IC scattering operates on "seed" photons on conserving their number while upgrading their energy by another factor $\gamma_{p}^{2}$, so as to yield photon energies $h \nu \sim h \nu_{s} \gamma_{p}^{2}$ and related luminosities $L_{C} \propto L_{\text {seed }} R n \gamma_{p}^{2}$.

The simplest source structure envisages as seeds the very $\mathrm{S}$ photons that are radiated by a single population of relativistic electrons in one, homogeneous zone of the jet, so as to yield also a beamed flux of gamma rays $L_{C} \sim L_{S} n R \gamma_{p}^{2}$. Such a synchrotron self-Compton (SSC) radiation process (e.g., Maraschi, Ghisellini \& Celotti, 1992) is sufficient to account also for the gamma-ray outputs in tight correlation with $\mathrm{O}$ - $\mathrm{X}$ as featured by many $\mathrm{BL}$ Lacs in their quiescent states.

An interesting development is constituted by growing hints or signs of apparently recurrent emissions in a number of Blazars, BL Lacs in particular. On the origin and modeling of these phenomena we will focus our attention in the present paper. 


\section{A Binary Driver of Recurring Jet Instabilities}

Interest in binary, massive BHs in AGNs started with Begelman et al. 1980, and is currently being spurred by the recent detections of bursts of gravitational waves constituting the events GW 150914 (Abbott et al. 2016a) and GW 151226 (Abbott et al. 2016b). These events have been shown to originate from binary systems of BHs with intermediate masses $M \sim 50 M_{\odot}$. The pairs have been caught in their late inspiral stages, as they were accelerating their orbital motions under increasing energy loss in gravitational waves to end up into catastrophic coalescence as predicted. In fact, extensive numerical work concerning binary BHs long pre-dated those events, aimed at including electromagnetic outputs from systems composed of two BHs and a warm disk or ring of gas (see Palenzuela et al. 2010, and the review by Baumgarte \& Shapiro 2011). Similar events are expected also in binary systems of two SMBHs (see Colpi 2014, Volonteri et al. 2015).

Ever as such emissions are dramatically enhanced during the final coalescence, they are numerically found to set in gently at much lower rates during the early, long inspiral stage. Similar computations can be focused as well on investigating the initially slow evolution of nearly Keplerian orbits and their interactions with the accretion disk; we aim at understanding non-linear light curve shapes recurring on scales of a few years in the e.m. emissions, as suggested by current gamma-ray observations discussed in Sects. 3 and 4.

The emissions of all Blazars are governed by the hydrodynamic and magnetic properties of the jet's relativistic plasma outflow (see Sect. 1). Jet formation and launching in many such sources appear to persist on gross average over timescales of many years. The considerable short term variations observed in their radiative outputs have often been considered to be fully erratic (see, e.g., Kelly et al. 2009). Jet formation and its short term instabilities are then attributed to random variability in the boundary conditions occurring at the base, such as accretion rate; alternatively, disk instabilities (see Nixon \& King 2015, and refs. therein) have been computed and discussed. In the present paper we consider an additional possibility.

We will investigate the binary SMBH scenario depicted in Fig. 1, where the primary launches a main jet that dominates the observed fluxes, but is perturbed by the secondary to a considerable degree, This we shall compute from considering the binary dynamics of a system with a total mass of $\sim 10^{8} M_{\odot}$, mass ratio $M_{2} / M_{1} \sim 10^{-1}$ and a current orbital size around several milli-pc. Specifically, the bulk properties of the main jet are affected by episodes of shaking, squeezing and twisting caused by differential gravitational and electromagnetic stresses along the non-circular, or even not simply Keplerian orbits. The results include timemodulations of the average strength $B(t)$ of the magnetic field, coordinated with changes in the local topology of B-lines by reconnecting and rearranging those that angularly diverge 


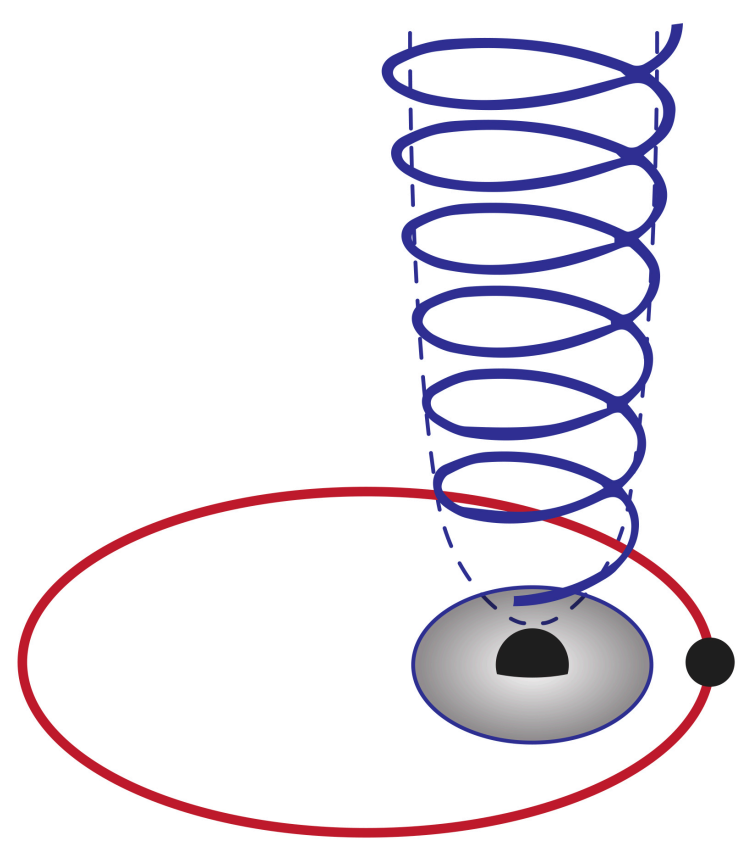

Fig. 1. - A schematic of our model for the SMBH binary system underlying a BL Lac Blazar. The primary $\mathrm{SMBH}$ produces a relativistic jet towering well above its formation region in the inner accretion disk (boundary marked by the thin curve). A SMBH companion orbits around the system barycenter close to the primary, following the red orbit. The gravitational force affects the jet base during its passes close to the periastron, modulating its emissions to a pattern with high peaks followed by long relaxations. The orbital size is of order $10^{16} \mathrm{~cm}$ for a total mass around $\sim 10^{8} M_{\odot}$, mass ratio $M_{2} / M_{1} \sim 10^{-1}$, with an intrinsic period $T \simeq 1.5$ years. 
across narrow layers, as we shall discuss in the next Section.

Here we just note that passive geometrical effects of gentle beam redirection along steadily curved or helical jets as advocated, e.g., by Raiteri et al. (2015) and discussed by A15 may be re-interpreted in terms of initially mild and slow dynamical effects that take place during the quasi-period. Our discussion will focus instead onto the dynamical driving in the jet of sites suitable for magnetic reconnections to take place.

\section{Unstable Jets}

We consider how the progression from large-scale jet instabilities proceeds through an intermediate stage of tearing perturbations to end up into kinetic effects that spread out electron acceleration to high energies.

We base our discussion on the canonical SSC radiation process recalled in Sect. 1. As to the primary $\mathrm{S}$ emission, we adopt standard values of the average magnetic field $B \sim 10^{-1}-1$ $\mathrm{G}$ in the source frame, at the height of some $10^{17} \mathrm{~cm}$ above the primary SMBH. These are modulated in time by factors of about three on timescales of a few years by compressing and bending $\mathrm{B}$ as discussed below.

The other basic component for $\mathrm{S}$ emission is constituted by highly relativistic electrons. Their acceleration occurs in regions within the jet that are affected by magnetic field lines packed, sheared and reconnected so as to induce macroscopic and kinetic effects. For a review covering the magnetic reconnection (MR) theory and the ongoing numerical simulations see Kagan et al. (2015), also Melzani et al. (2014). For recent theoretical developments on reconnecting structures in collisionless plasmas, see Coppi (2016); for detailed observational results concerning reconnections in a magnetospheric environment, see Burch et al. (2016).

The intermediate output of MRs and associated tearing instabilities is constituted by strings of "magnetic islands" or plasmoids, many of which then merge into a few giant ones. Meanwhile, on the kinetic side sharply sheared, or even annihilating B-lines induce strong if confined E-lines; these repeatedly and efficiently accelerate electrons in situ to high values of $\gamma \sim 10^{3}$, particularly around the giant, coalescing plasmoids and in the intervening gaps. Next, we focus on the key parameters governing these two, related sides of the processes occurring in magnetized jets. 


\subsection{The macroscopic side}

The relevant parameter for stability on large scales is provided by the bulk magnetization of the jet

$$
\sigma_{j}=\frac{\bar{B}^{2}}{4 \pi n_{p} m_{p} c^{2} \Gamma^{2}} .
$$

This depends on the average field that appears in the magnetic stress $\bar{B}^{2} / 4 \pi$, and on the kinetic stress $n m_{p} c^{2} \Gamma^{2}$ dominated by the jet protons (see Celotti \& Ghisellini 2008).

The condition $\sigma_{j} \lesssim 1$ yields stability of a jet on large scales, for example, on the scales $10^{16}-10^{17} \mathrm{~cm}$ numerically investigated by, e.g., Mignone et al. (2013); on the other

hand, values $\sigma_{j} \gtrsim 1$ are found by the same authors to promote jet instabilities. Thus the magnetization $\sigma_{j}$ constitutes the main parameter governing the overall stability of a jet configuration on large scales.

Using values of $B \sim(0.1-1) \mathrm{G}, n \sim 10^{-1}-10^{2} \mathrm{~cm}^{-3}$ related to the accretion rates on the primary SMBH, and values $\Gamma \sim 10$, as derived, e.g., by Paggi et al. (2009), Tavani et al. (2015), and observed by Hovatta et al. (2009), we find $\sigma_{j}<10^{-1}$ to hold in FSRQs, while $\sigma_{j} \lesssim 1$ prevails in BL Lacs. Thus the latter appear to sit on the brink of instability, and may be driven unstable by minor dynamical perturbations such as may be induced by a binary BH companion. On evaluating at T/10 (details are given in the Appendix) the period fraction with strong primary acceleration while revolving around the barycenter, it is seen that the jet cannot remain connected over scales $\ell>c T / 10 \sim 10^{17} \mathrm{~cm}$ against dynamical perturbations that shake its base on an effective timescale of a few months. This also sets an upper limit to any spread along the axis of the sources of correlated emissions.

\subsection{The kinetic side}

At the opposite extreme constituted by the local kinetic level, acceleration of electrons to high energies is governed by repeated MR events. The acceleration process has been parametrized and numerically computed in terms of the electron local magnetization (see the review of Kagan et al. 2015)

$$
\sigma_{e}=\frac{B^{2}}{4 \pi n_{e} m_{e} c^{2}},
$$

which depends on the local magnetic field $B$ and electron density $n_{e}=n_{p}$, given their mass $m_{e}$.

Many numerical calculations have been carried out in the range of $\sigma_{e}$ values from tens 
to up to several hundreds (see Sironi \& Spitkovsky 2014, Melzani et al. 2014). Remarkably, for increasing $\sigma_{e}>10^{2}$ the resulting electron energy distributions approach flat power-laws $n(\gamma) \propto \gamma^{-1.5}$ up to, or asymptotically exceeding $\gamma \sim 10^{3}$ limited by computing times (e.g., Melzani et al. 2014). We stress that in the simplest case the relation

$$
\sigma_{e} \gg \frac{m_{p}}{m_{e}} \sigma_{j} \sim 10^{3} \sigma_{j}
$$

holds, so that the electron energies can grow to values $\gamma \sim 10^{3}$ and possibly beyond as soon as bulk instability conditions are established by values ${ }^{1}$ of $\sigma_{j}>1$. In other words, conditions conducive to bulk instability act as a trigger for strong particle accelerations to occur. Note, however, that the electron magnetization in Eq. 2 affects the particle energy distribution function only in the presence of topological configurations of the local magnetic field that include sheared layers or close reversals conducive to tearing and reconnections in the jet's collisionless plasma.

Extensive and detailed computations have recently been presented by Yuan et al. 2016 concerning the link between macroscopic field configurations with higher magnetization and kinetic conditions conducive to acceleration. We conclude that a slow time shift of macroscopic conditions toward increased $\sigma_{j}$ and sheared $\mathbf{B}$ topology can end up into triggering and enhancing local accelerations governed by high values of $\sigma_{e}$.

\subsection{The binary drive}

Thus current knowledge backs our expectation that in BL Lac jets - by themselves in near-critical conditions - even modest perturbations that increase B can easily drive the structure beyond the threshold of macroscopic instabilities. Soon after, the conditions will progress toward strong kinetic instabilities if the macroscopic modes induce local torsion and shear of $\mathbf{B}$. The ensuing scale for field shearing will be close to the longitudinal mode wavelengths experienced by the jet, that are of order of $10^{16}-10^{17} \mathrm{~cm}$ as numerically computed, e.g., by Mignone et al. 2013 (see also Striani et al. 2016).

In the case of a binary SMBH system, we envision as driving agent the dynamical perturbations of the magnetic jet configuration by the companion orbiting around the accretion disk, from which the main jet is launched. When the perturbations occur, the field $\mathbf{B}$ not

\footnotetext{
${ }^{1}$ Note that Eq. 3 as it stands applies for values of $\gamma \lesssim m_{p} / m_{e}$. For larger values, the electron contribution to the total kinetic energy should not be neglected; $\sigma_{j}$ is correspondingly decreased, and so is the effective $\sigma_{e}$. This feature may imply self-regulation to limiting energies $\gamma \sim 10^{4}$.
} 
only is locally compressed to larger strength $B+\delta B \simeq$ a few times $B$, but also is locally sheared so that regions of sharply different magnetic field lines are drawn together.

In a nutshell, binary perturbations drive macroscopic jet instabilities that offer many suitable locations for MR to occur and trigger effective electron acceleration; the ensuing emissions are detailed next.

\section{Emissions and Their Interpretations}

The basic feature to be expected from the binary drive of jet instabilities as discussed in Sect. 3 concerns a recurring, non-linear spike-trough pattern in the light curves, reflecting the stroke-relaxation nature of the dynamical drive along a Keplerian elliptical orbit.

\subsection{Light curves}

To be specific, we shall refer to the BL Lac object PG $1553+113$ as an interesting candidate for recurrent non-linear gamma-ray emission as observed and discussed by Ackermann et al. (2015, henceforth A15). The source features in its continuously monitored gammaray emission high amplitude modulations that recur with an apparent period $P \simeq 2.2 \mathrm{yr}$; at the source redshift $z \simeq 0.5$ (see Danforth et al. 2010) this corresponds to an intrinsic value $T \simeq 1.5 \mathrm{yr}$. Three full (in fact, 3.5), consecutive periods are continuously covered by the existing gamma-ray observations of PG $1553+113$ presented in A15; these data extend the dynamic range (number of cycles and amplitudes) of previous blazar periodicity analyses such as that of Vaughan et al. 2016. From the A15 analysis of PG 1553+113, a next gamma-ray peak is expected in early 2017.

In the Appendix we derive in detail a simple model for the gamma-ray light curve based on the gravitational modulation in a binary system, as impressed by the companion on the primary $\mathrm{BH}$ and its associated jet. Our result is presented here in Fig. 2, along with the 7-year data concerning PG 1553+113 from A15. It is seen that our model yields a non-linear pattern of the light curve with prominent peaks and wide troughs, that for an eccentricity $\epsilon \simeq 0.2$ is quantitatively very close to the overlaid data points. 


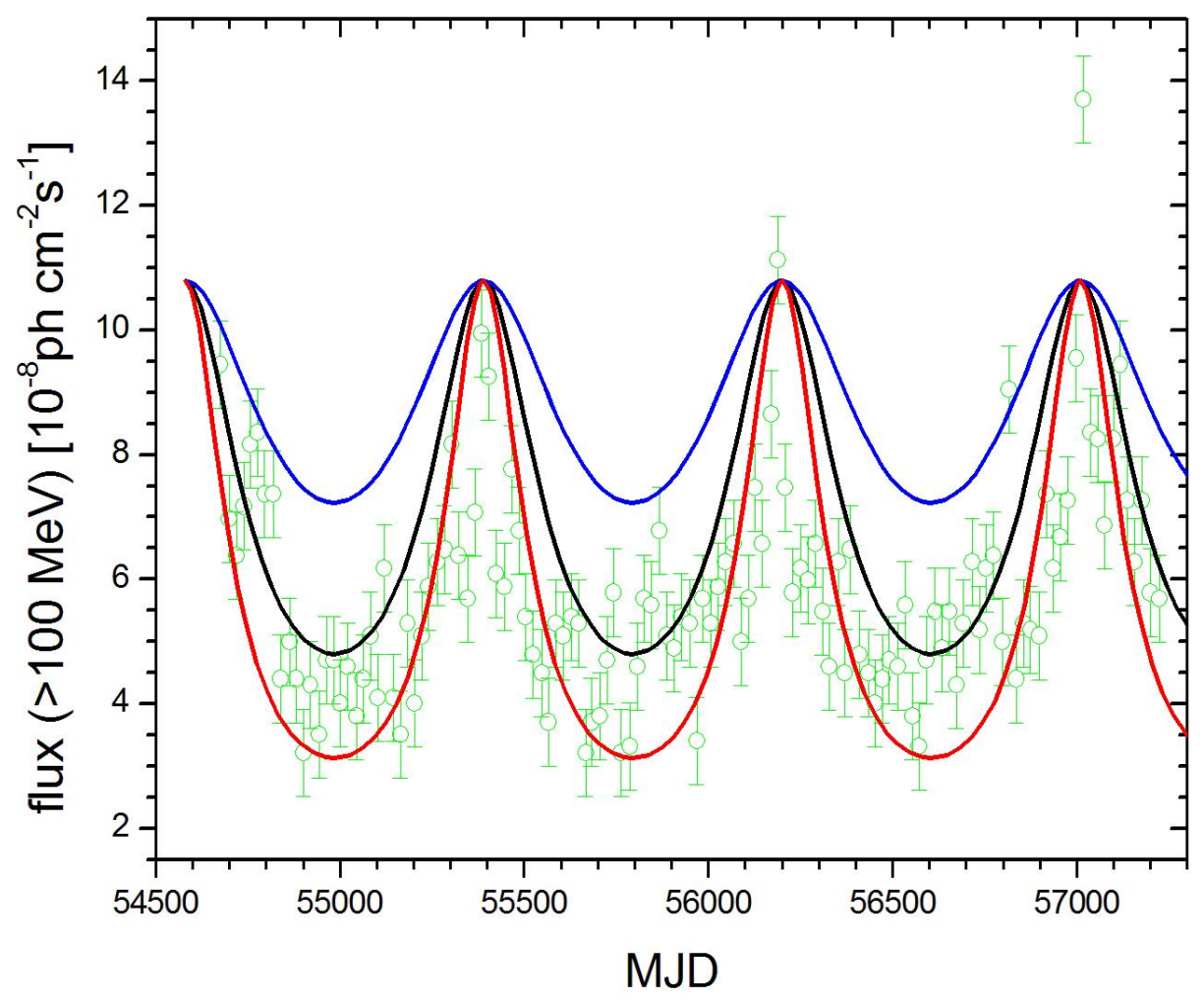

Fig. 2. - The gamma-ray light curve of PG 1553+113 (green data points and error bars from A15) is compared with the predictions from our model, in which the gamma-ray flux is proportional to the gravitational force after $f(t) \propto F(r) / F\left(r_{\max }\right)$ (cf. Eq. A1 in the Appendix) along the elliptical orbit at a distance $r$ from the focus. We used the apparent period $P=2.2$ yr (see Sect 4.2 ), and three values for the eccentricity: $\epsilon=0.1$ (blue curve), $\epsilon=0.2$ (black curve) and $\epsilon=0.3$ (red curve). For $\epsilon=0$ the orbit would be circular and the light curve just flat, apart from minor random fluctuations. 


\subsection{Spectral distributions}

The basic spectral change expected from compressed and sheared magnetic fields in the jet is twofold. To begin with, we expect an increased overall SSC emissivity, led by the optical S emission and followed after some weeks by IC radiation at $\mathrm{GeV}$ energies. Such a direct correlation is well compatible with the data as discussed by A15, but is also expected to be appreciably smeared out along the jet axis by outflow and long cooling times for both component sources (see Table 1 and its caption).

In detail, the implied enhancements of S, optical radiation and of its IC counterpart at $\mathrm{GeV}$ energies are shown by the spectra marked by red color in Fig. 3 with overlaid data. Such flux variations in both the R-band and the GeV component range by a factor around 3 from minima to maxima. In conclusion, the emissions in the optical, the soft $\mathrm{X}$ and the $\mathrm{GeV}$ bands appear to generally correlate along the spectra in shape and timing in the way described by the canonical SSC radiation model.

On the other hand, the behavior at keV energies of PG 1553+113 appears to stray outside the above framework, and has been reported as "enigmatic" (see, e.g., Raiteri et al. 2915). For one, the increases during episodes of optical rise differ in softer X-ray ranges: they rise from a factor of 3 in the range $0.3-2 \mathrm{keV}$, but are up to factors $5-10$ in the 2-10 $\mathrm{keV}$ range as reported by Aleksic et al. (2015) for the episode around MJD 56000. For two, other BL Lacs such as Mkn 421 appear to share with PG 1553+113 such keV features as missing correlation with neighboring $\mathrm{X}$ rays, and positive correlations with $\mathrm{TeV}$ emissions (Balokovic et al., 2016).

\subsection{An additional spectral component in $\mathrm{X}$ rays}

To interpret such a behavior, we recall the contents of Sect. 3. In particular, in Sect. 3.1 we noted that a BL Lac jet is normally metastable, so that a limited increase of $B$ can drive it first toward macroscopic, and then into kinetic instability conditions.

In a first stage the near-critical bulk magnetization $\sigma_{j} \lesssim 1$ is increased by a factor of a few, as deduced from the optical light curves enhanced during their 100-day rise. This implies a drift from marginal values of $\sigma_{j}$ into the fully critical range for macroscopic instabilities. If these produce twisted and inverted $\mathbf{B}$ lines a second stage arises, driven by increasing local magnetizations $\sigma_{e} \propto \sigma_{j}$ after Eq. 3. These enhance MRs and cause extensive particle acceleration. Based on current numerical simulations that are exploring different regimes of ion-electron plasmas (see Melzani et al. 2014, Kogan et al. 2015), we derive that the maximal electron energies can approach values $\gamma_{p} \sim 10^{4}$ for values of $\sigma_{e}$ exceeding $10^{2}$. 
Table 1: parameters for modelling the spectral states of PG 1553+113.

\begin{tabular}{|c|c|c|c|c|c|c|c|c|c|}
\hline State & Comp. & $\boldsymbol{\Gamma}$ & $\mathbf{B}(\mathbf{G})$ & $\mathbf{R}(\mathbf{c m})$ & $\mathbf{K}\left(\mathbf{c m}^{-3}\right)$ & $\gamma_{\mathbf{b}}$ & $\gamma_{\mathbf{m i n}}$ & $\alpha_{\mathbf{1}}$ & $\alpha_{\mathbf{2}}$ \\
\hline low & gray & 15 & 0.25 & $4.0 \cdot 10^{16}$ & 0.6 & $9 \cdot 10^{3}$ & $4 \cdot 10^{3}$ & 2 & 3.9 \\
\hline enhanced soft & red & 15 & 0.29 & $4.0 \cdot 10^{16}$ & 0.6 & $9 \cdot 10^{3}$ & $3 \cdot 10^{3}$ & 2 & 3.9 \\
enhanced hard & blue dashed & 15 & 0.29 & $3.5 \cdot 10^{16}$ & 0.1 & $3 \cdot 10^{4}$ & $5 \cdot 10^{3}$ & 2 & 4.3 \\
\hline
\end{tabular}

The Table summarizes the parameters used in our SSC spectral modelling of PG 1553+113 (see Fig.

3.) The Table provides values for the bulk Lorentz factor $\Gamma$, the average magnetic field $B$ inducing Synchrotron emission, the source size $R$, the electron energy distribution $n(\gamma)=K \gamma_{b}^{-1}\left(\gamma / \gamma_{b}\right)^{-\alpha}$ with normalization $\mathrm{K}$, upper break at $\gamma_{b}$ and lower bound $\gamma_{\text {min }}$, and power-law indices $\alpha_{1}$ below and $\alpha_{2}$ above the break. The associated cooling times for $\mathrm{S}$ and for IC (see Rybicki \& Lightman 1979) $\mathrm{read} t_{c} \simeq 2 R / c \simeq 30 \mathrm{~d}$.

Such enhanced accelerations will add a stronger and harder spectral component in the $\mathrm{X}$-ray range around and above $1 \mathrm{keV}$, as indeed detected in at least two episodes within the currently available data concerning PG 1553+113 (A15). The X-ray flux increase amounts to factors of 5-10, i.e., substantially larger than the related rise of optical and GeV emissions. Clearly, here one-zone, single population modelling of the X-ray emission together with the optical and $\mathrm{GeV}$ emissions would not be adequate. Our picture, on the other hand, implies additional particle acceleration triggered in nearby zones with increasing values of $\sigma_{e}$. The corresponding SEDs for one of such episodes are presented in Fig. 3.

\section{Discussion and Conclusions}

We have argued in Sect. 2 that dynamic events in Blazar jets - and specifically in BL Lac Objects - originate from an underlying binary system of two Super Massive Black Holes with total mass $M \sim 10^{8} M_{\odot}$. Binary dynamics constitutes, as we stated in Sect. 2 and explained in Sect. 3, the prime mover for a chain of events in the jet: it recurrently drives bulk plasma instabilities in the metastable magnetized jet launched by the primary; in turn, these trigger local tearing instabilities and in situ electron acceleration to high energies corresponding to, and at times exceeding $\gamma \sim 10^{3}$.

Such local instabilities and accelerations are known to arise in narrow layers of a collisionless plasma, where reconnections occur among adjacent $B$-lines that are strongly sheared or even reversed, as we detailed in Sects. 3.1 and 3.2. Such processes are bound to be triggered during dynamic episodes of large-scale squeezing and shearing of a magnetized jet. A conducive context for such episodes to occur is provided by binary SMBH dynamics (as 


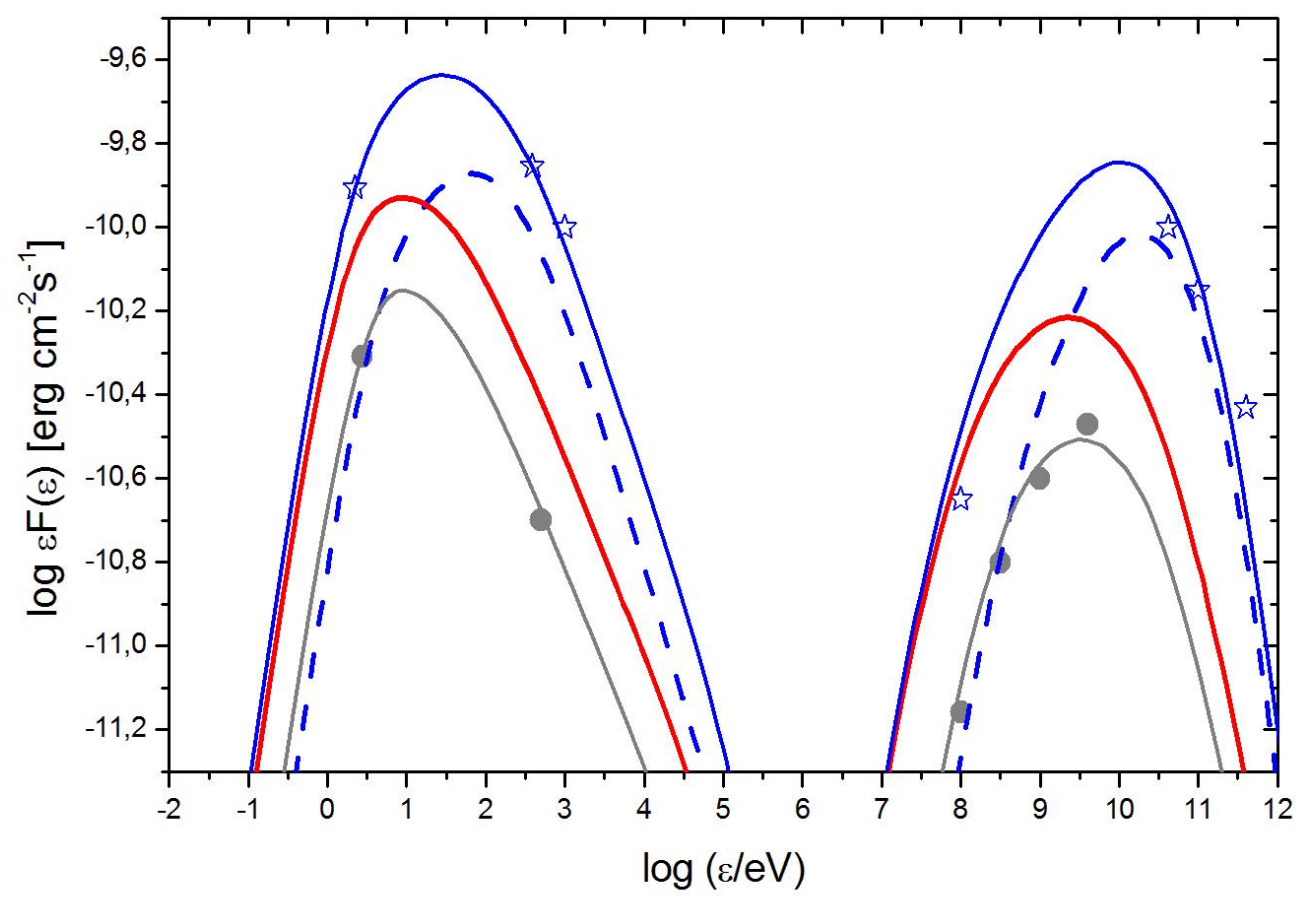

Fig. 3.- Spectral energy distribution (SED) of the emissions by electrons accelerated in the jet of the BL Lac PG 1553+113 . Spectral data are from Ackermann et al. (2015) and Aleksic et al. (2015). The gray line shows the spectral state during normal conditions. The blue, dashed curve represents the harder, increased Synchrotron and IC components resulting from the extra electron acceleration discussed in Sect. 4.3. The total emission in the enhanced state is represented by the blue solid curve. EBL de-absorption in the TeV data after Aleksic et al. 2012. 
discussed in Sect. 3.3), that also provides the simplest interpretation for the recurrent, nonlinear pattern of peaks and trough in the light curves of the BL Lac source PG 1553+113 as discussed in Sect. 4.1.

We have analyzed in Sect. 4.2 the different spectral states that arise in this source and exhibit repetition. They start with a recurrent increase of the optical Synchrotron and of the inverse Compton $\mathrm{GeV}$ radiations produced by the same population of energetic electrons. We have stressed in Sect. 4.3 the additional spectral component that has been observed in the $\mathrm{keV}$ range as the optical light curve approaches a peak. We traced it back to spreading accelerations triggered by additional reconnections stimulated by binary dynamics. We expect this component to correlate with $\mathrm{TeV}$ enhancement, as occurring in other BL Lacs.

So we conclude that at times two spectral states overlap in the emissions from PG 1553+113 . One is well represented as quasi-periodic emission, that envisages the optical - soft X-ray components and the GeV photons as linked components of the same SSC radiation process; the latter therefore should correlate with the former, after a lag of some weeks. The other, more energetically demanding state requires additional and higher electron accelerations that produce a harder X-ray peak, as a component (see Section 4.3) observed in two out of three monitored instances. this we expect to correlate with $\mathrm{TeV}$ enhancements, not necessarily with the optical $-\mathrm{GeV}$ radiations.

Next we summarize our overall conclusions, and specifically two points. First, we have described how a BL Lac jet, normally sitting on the brink of instability, can undergo recurrent stresses from the binary dynamics sufficient to stimulate cyclic emissions. In fact, increasing bulk magnetization leads to large scale instabilities that cascade down to small scale MRs and ultimately percolate to the kinetic level; there they accelerate the electrons that emit from the optical to the gamma-ray band, and sometimes beyond.

Second, the binary Keplerian motion drives strokes and allows relaxations corresponding to orbital arcs close to, and far away from the periastron, respectively. So it imprints a specific non-linearpattern of sharp peaks and wide troughs in the light curves at different frequencies; these are particularly sharp in gamma rays that have been continuously monitored from space. Such a pattern can provide diagnostics for orbital parameters, in particular for the orbital eccentricity $\epsilon$ as detailed in the Appendix, and used in Fig. 2 to derive for PG $1553+113$ a value $\varepsilon \simeq 0.2$.

As to predictions and prospects, in the framework of the next gamma-ray peak expected for PG 1553 in early 2017 by A15, we stress the following points. (1) The new peak should appear in reasonable phase agreement (within the time binning) with the previous three occurrences. (2) Thus the optical emission should be monitored as continuously as possible 
across the peak phase range, with the help of telescopes strategically coordinated. The optical emission may be quite structured as shown by Figs. 1 and 2 of A15; in our picture we expect the main optical increase to begin several days to some weeks before the smoother and better sampled gamma-ray peak, which can sound a last call (as it were) for aimed X-ray observations. (3) In fact, the X-ray extra component in the keV range is expected to be triggered by expanding reconnections that occur just at, or on the approach to the periastron. Current data have the peak of additional X-ray emission corresponding in one case to the gamma-ray peak near MJD 57000 (to within the time binning); in another case, preceding he gamma-ray peak by $\sim 100$ days. Our model contemplates enhanced keV emission somewhere between the rise of the optical and of the gamma-ray light curves, on account of the spread along the jet of the S and the IC main sources implied by their long cooling times (see Sect. 4.3, and Table 1).

It is interesting to recall that a similar radiative behavior to PG $1553+113$ is shared by other BL Lacs, in particular by Mrk 421 with its long puzzling, X-ray outbursts correlated with $\mathrm{TeV}$ activity but not with optical and GeV emissions (se Raiteri et al. 2015, Balokovic et al. 2015). Some of these sources (listed, e.g., by Sandrinelli et al. 2014) have been proposed also as candidates for recurring behavior in the optical and gamma-ray bands.

We note that the dominance of possible BL Lacs' periodicities over FSRQs' in the set of current candidates may just reflect selection favoring the former on account of their generally smaller redshifts and lack of disturbing $O-U V$ background from BLs and BBB pointed out in Sect. 1. On the other hand, intrinsic association of BL Lacs with binary sources is suggested by a number of circumstances. First, as a consequence of hierarchical galaxy formation, the BL Lacs as aged AGNs (Cavaliere \& D'Elia 2002, Böttcher \& Dermer 2002) must have witnessed several merging events involving their host nuclei. Thus, expanding on the pioneering suggestion by Begelman et al. (1980) such nuclei would be more massive and likely to have accrued several SMBHs to eventually form a dominant binary pair. In addition, the intrinsic meta-stability of BL Lac jets (see Sect. 3) makes the latter susceptible even to minor dynamic disturbances in forming a recurrent binary-driven source. Furthermore, the absence in BL Lac spectra of thermal features as recalled above points toward gas-poor conditions in the vicinity of the central SMBH binary; these prevent local energy dissipations from occurring, and allow for longer binary lifetimes implying higher intrinsic statistics (see Colpi 2914 and refs. therein), limited mainly by e.m. losses, and eventually cut off by a gravitational catastrophe (see Sect. 2).

Finally, we consider the A15 prediction concerning a next maximum of PG $1553+113$ in early 2017, with a spread of some 20-40 days due to time binning; this would mark an obvious plus for the periodic view. On the quantitative side, extending the sequence of fully 
observed cycles from 3 to 4 would considerably strengthen the confidence level 0.99 recalled in Sect. 4. Thus by early 2017 one had better be prepared with astrophysical analysis of the currently known cycles as undertaken here, and with coordinated observations of keV $-\mathrm{TeV}$ emissions. We add that evidence of any secondary jet would provide a tell-tale signature of binarity that deserves a close search, despite a number of difficulties: the expected weak power ratio $\propto M_{2} / M_{1} \sim 10^{-1}$ even in the favorable case of both jets fed by accretion at a common fraction of their Eddington rates; the uncertainty about the jet relative angle; and the range in the time phase caused by precessional shifts.

These tasks are demanding, but the reward of confirmed evidence for binarity would be worth of keen observational efforts. It is genarally agree that understanding even a single SMBH binary would open a new window on the vexing issue of co-evolution of galaxies and their central BHs (see Lapi et al. 2014, Volonteri et al. 2015). In addition, a few confirmed SMBH binaries with assessed orbital parameters such as proposed here may be of keen value for planning searches of giant, slow bursts of gravitational waves with space interferometers such as eLISA (see eLISA Consortium, 2013). In fact, the path from host galaxy mergers to a sub-pc binary doomed to eventually coalesce, is complex (see Colpi 2015) and currently still fraught with a number of uncertain steps ("stalling" in particular), that not even massive numerical computations or simulations have been able to fix to now.

We are indebted to Bruno Coppi for enlightening discussions on magnetic field reconnecting structures in collisionless plasmas. We thank our referee for recommending a generally conservative attitude towards the quasi-periodicity of PG 1553+113), and for pressing us to specify the role of light curve profiles, as we did in the revised MS. Work partially supported by the ASI grant no. I/028/12/2. 


\section{References}

Abbott et al., 2016a, Phys. Rev. Letters, 116, 061102

Abbott et al., 2016b, Phys. Rev. Letters, 116, 241103

Ackermann, M., Ajello, M., Albert, A., et al., 2015, ApJL, 813, 41 (A15)

Aleksic J., Alvarez, E.A., Antonelli, L.A. et al., 2012, ApJ, 748, 46

Aleksic J., Ansoldi, S., Antonelli, L.A. et al., 2015, MNRAS, 450, 4399

Balokovic, M., Paneque, D., Madejski, G. et al., 2016, 819, 156

Baumgarte, T.W. \& Shapiro, S.L., 2011, Physics Today, 64, 32

Begelman, M., Blandford, R.D., \& Rees M.J., 1980, Nature, 207, 307

Boettcher, M. \& Dermer, C.D., 2002 ApJ, 564, 86

Burch, J.L., Torbert, R.B., Phan, T.D. et al., 2016, Science, 352, 1189

Cavaliere, A. \& D'Elia, V., 2002, ApJ, 571, 226

Celotti, A \& Ghisellini, G., 2008, MNRAS, 385, 283

Cohen, D.P, Romani, R.W., Filippenko, A.V., et al, 2014, ApJ, 797, 137

Colpi, M., 2014, Space Science Reviews, 183, 189

Coppi, B., 2016, Plasma Phys. Rep., 42, 383

Danforth, C.W., Keeney, B.A., Stocke, J.T., Shull, J.M., Yao, Y., 2010, ApJ, 720, 976

eLISA Consortium, 2013, The Gravitational Universe, arXiv:1305.5720v1

Ghisellini, G., 2016, arXiv:1609.08606

Hovatta, T., Valtaoja, E., Tornikoski, M., Lahteenmäki, 2009, A., 2009, A\&A 494, 527

Kagan, D., Sironi, L., Cerutti, B., Giannios, D., 2015, SSRv, 191, 545

Kelly. B. C., Bechtold, J. \& Siemiginowska, A., 2009, ApJ, 698, 895

Krawczynski, H., Hughes, S.B., Horan, D., et al., 2004, ApJ, 601, 151

Landau, L. \& Lifshitz, E.M., 1976, Mechanics, Oxford, Pergamon Press

Lapi, A., Raimundo, S., Aversa, R., et al., 2014, ApJ, 782: 69 
Maraschi, L., Ghisellini, G. \& Celotti, A., 1992, ApJ, 397, L5

Melzani, M., Walder, R., Folini, D., Winisdoerffer, C., Favre, J.M. et al., 2014, A\&A, 570A, 111

Mignone, A., Striani, E., Tavani, M., and Ferrari, A., 2013, MNRAS, 436, 1102

Nixon, C., \& King, A., 2015, arXiv:1505.07827

Palenzuela, C., Garrett, T., Lehner, L., Liebling, S.L., 2010, PhRvD, 82, 404

Peterson, B.M., 1997, An Introduction to Active Galactic Nuclei, Cambridge Univ. Press

Peterson, B.M., 2006, The Broad-Line Region in Active Galactic Nuclei, Lect. Notes Phys. 693, 77 (Springer)

Raiteri, C. M., Stamerra, A., Villata, M., et al., 2015, MNRAS, 454, 353

Rybicki, G.H., Lightman, A.P., 1979, Radiative Processes in Astrophysics (Wiley)

Sandrinelli, A., Covino, S., Treves, A. 2014, A \&A, 562, 79

Sironi,L. \& Spitkovsky, A., 2014, ApJL, 783, 21

Striani, E., Mignone, A., Vaidya, B., Bodo, G., Ferrari, A., 2016, MNRAS, 462, 2970

Tavani, M., Vittorini, V., Cavaliere, A., 2015, ApJ, 814, 51

Vaughan, S., Uttley, P., Markowitz, A.G., et al., 2016, MNRAS, 461, 3145

Volonteri, M., Bogdanovic, T., Dotti, M., Colpi, M., 2015, Proc. XXIXth IAU General Assembly, arXiv:1509.09027v1

Yuan, Y, Nalewaiko, K., Zrake, J., East, W. E., Blandford, R., 2016, ApJ vol. 828; also preprint arXiv:1604.03179. 


\section{A. Appendix: Deriving the Light Curves and the Corresponding Orbital Eccentricities}

In Sect. 3 we consider a gravitationally bound binary system with separation of several milli-pc. Drawing on time-honored dynamical relations holding for Keplerian, elliptical orbits of the secondary (see, e.g., Landau \& Lifshitz 1976, and their Figure 11) compared with the light curves observed by A15 (cf, their Figs. 1, 2), we detail how we deduce our expected light curve and the corresponding orbital eccentricity $\varepsilon$.

We shall follow two ways. For semplicity, in both we neglect the primary motion around the system's center of mass, and in particular the size of its obit that scales as $M_{2} / M_{1} \lesssim 10^{-1}$. We base on the gravitational stress from the secondary being proportional to the force between the two BHs, with enhanced emission also proportional to it.

1) The ratio of the force at periastron to that at the opposite vertex reads

$$
F_{\text {max }} / F_{\text {min }}=(1+\varepsilon)^{2} /(1-\varepsilon)^{2} .
$$

In our approach, this also yields the ratio of gamma-ray fluxes at maxima and minima.

From the gamma-ray light-curve in A15 (their Fig. 1) we read out the observed value $\simeq 3$ for the flux ratio between peaks and troughs. After Eq. 4 this corresponds to an orbital eccentricity $\varepsilon \simeq 0.25$.

In addition, in Fig. 4 we plot the full dependence $f(t) \propto F[r(t)]$ of flux that we expect along the orbit at a distance $r(t)$ from the focus, parameterized with three eccentricity values: $\epsilon=0.1,0.2,0.3$. The data are overplotted, and agreement is found for $\epsilon=0.2$

2) A second approach considers the time fraction along the orbit most affected by such an effect. For an estimate, we concentrate on the points on the elliptical orbit marking the intersections with the so-called latus rectum $p$, i.e., the orbital distance corresponding to the "true anomaly" angle $\phi=\pi / 2$, with $\phi=0$ at periastron. The time $\Delta t$ spent on the orbital arc circling the focus from $p$ through the periastron out to the opposite phase, is given by

$$
\frac{\Delta t}{P}=\frac{\arccos (\varepsilon)-\varepsilon \sqrt{1-\varepsilon^{2}}}{\pi} .
$$

Fig. 4 shows the ratio $\Delta t / P$ as a function of orbital eccentricities. From the observations recalled above we see that the strongly enhanced emission lasts for $\sim 280$ days, corresponding to a fraction $\Delta t / P \simeq 0.35$. Interpreting this feature as caused by the gravitational perturbation of a secondary $\mathrm{BH}$ in an elliptical orbit, we deduce from Fig. 4 a value of the eccentricity $\varepsilon \simeq 0.23$. 
Thus we conclude that on interpreting the repetitive pattern of enhanced high-energy emissions from PG $1553+113$ in terms of stresses induced by binary dynamics, both the above approaches lead to evaluate for the orbital eccentricity a value close to $\varepsilon=0.2$.

Note that for a circular orbit the expected light curve is just flat; in fact, from Eq. 4 one obtains $F_{\max } / F_{\min }=1$. Concerning Fig. 2 in the main text, we stress that the dynamically stimulated (as it were) emission maxima largely exceed the spontaneous random fluctuations best noticed in the troughs. 


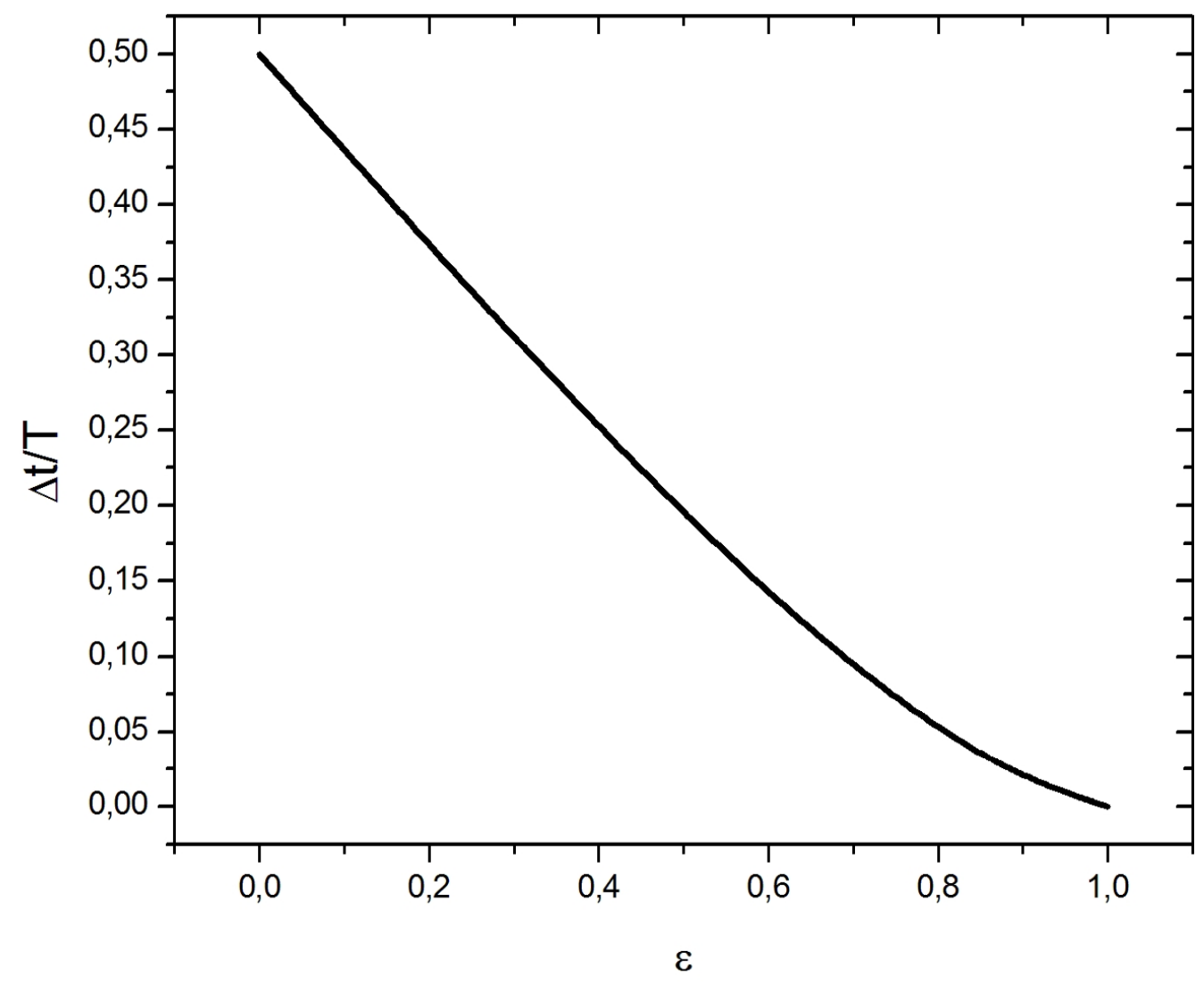

Fig. 4. - The ratio $\Delta t / P$ plotted as a function of the eccentricity for a Keplerian elliptical orbit. This quantity marks the strongly active fraction of the orbital period, that we take to be the interval between the intersections of the "latus rectum", each side of the periastron. 